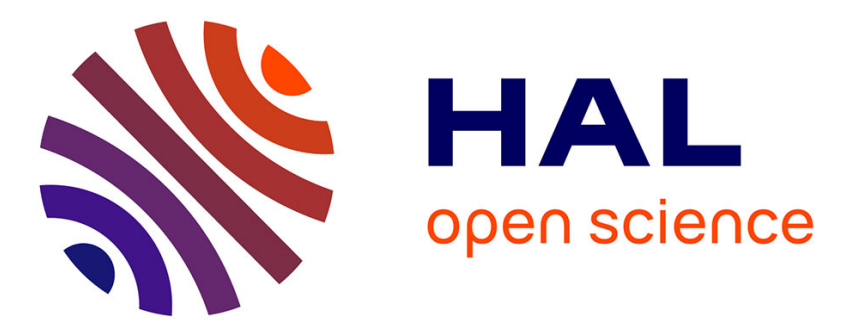

\title{
The monolithic suspension for the Virgo interferometer
} M Lorenzini

\section{To cite this version:}

M Lorenzini. The monolithic suspension for the Virgo interferometer. Classical and Quantum Gravity, 2010, 27 (8), pp.84021. 10.1088/0264-9381/27/8/084021 . hal-00587617

\section{HAL Id: hal-00587617 https://hal.science/hal-00587617}

Submitted on 21 Apr 2011

HAL is a multi-disciplinary open access archive for the deposit and dissemination of scientific research documents, whether they are published or not. The documents may come from teaching and research institutions in France or abroad, or from public or private research centers.
L'archive ouverte pluridisciplinaire $\mathbf{H A L}$, est destinée au dépôt et à la diffusion de documents scientifiques de niveau recherche, publiés ou non, émanant des établissements d'enseignement et de recherche français ou étrangers, des laboratoires publics ou privés. 


\title{
The monolithic suspension for the interferometer Virgo
}

\author{
M Lorenzini ${ }^{1}$ on behalf of Virgo Collaboration \\ ${ }^{1}$ INFN, Istituto Nazionale di Fisica Nucleare, Sez. di Firenze via G. Sansone 1, \\ 50019 Sesto Fiorentino (FI), Italy \\ E-mail: lorenzini@fi.infn.it
}

\begin{abstract}
Monolithic fused silica suspensions are needed to reduce the suspension thermal noise level in future, ground-based gravitational waves interferometric detectors. We present the status of the monolithic suspension system which will be employed for the test masses of the Virgo+ detector. Two fully monolithic suspensions have been realized using a spare Virgo mirror, so the assembling pipeline was checked; moreover, a very reliable recovery procedure was developed, to allow an efficient and fast (about a week) suspension repairing in case of wires failure. The performances of a full scale prototype of the last suspension stage, suspending an aluminum dummy mass, were tested and the mechanical behaviour of the suspension is currently studied in vacuum. The obtained results, crucial to finalize the design of the silica suspension elements for the advanced version of the interferometer, are reported.

PACS numbers: $04.80 . \mathrm{Nn}, 43.40 . \mathrm{Cw}, 62.40 .+\mathrm{i}, 46.50 .+\mathrm{a}$
\end{abstract}

\section{Introduction}

Suspension thermal noise, coming from the thermal excitation of the mechanical degrees of freedom of the wires that suspend the test masses, is a limit to present day sensitivity of ground based gravitational waves interferometric detectors at low frequency. Virgo [1] is a $3 \mathrm{~km}$ long arm power-recycled Michelson interferometer located near Pisa (Italy). In Virgo, suspending elements are C85 steel wires, $0.2 \mathrm{~mm}$ thick, which pass below the mirrors in a cradle-like arrangement [2]. Spurious contact losses and the high structural loss of steel $\left(\phi \simeq 10^{-4}\right)$ make this solution unsuitable for future detectors. Fused silica has a very low loss level $\left(\phi \simeq 10^{-9}[3,4]\right)$ at room temperature and high tensile strength, thus it has been suggested as ideal material for the suspension wires. Fused silica wires, connected to the mirror in a monolithic arrangement, have been installed in the German-British GEO600 detector [5]; and now advanced ground based gravitational waves interferometric detectors, such as Advanced LIGO (USA) [6] and Advanced Virgo [7], are planning to install monolithic suspensions. The monolithic design for the "intermediate" advanced version of Virgo, called Virgo+[8], is being finalized and is detailed in the following sections. 


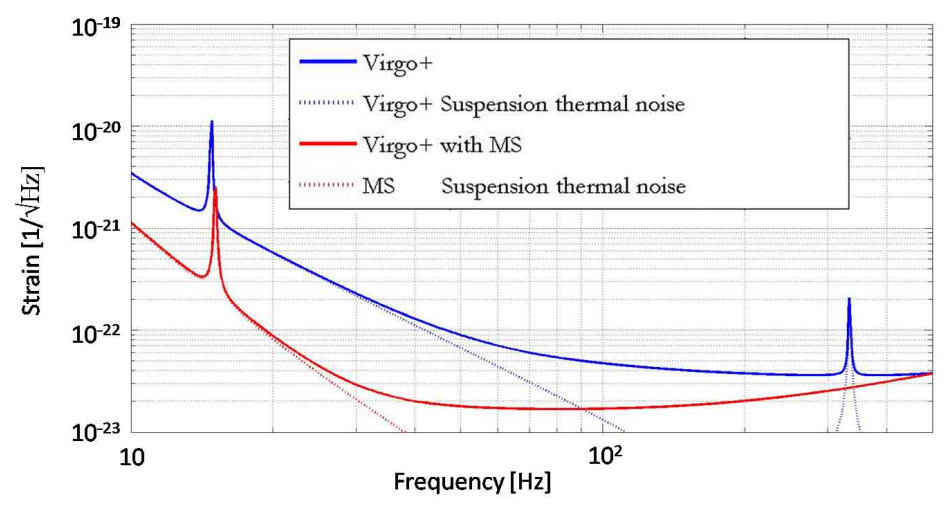

Figure 1. Sensitivity curves of Virgo+ in the frequency region where the suspension thermal noise contributions are predominant. It is evident the gain in sensitivity due to the MS (red curve) with respect to the present one (blue curve) with steel wires. For a more detailed description of the curve refer to [9].

\section{The monolithic suspensions for Virgo+}

Virgo is now in scientific data taking mode in coincidence with LIGOs. Due to the need of collecting as much scientific data as possible, the upgrade to Virgo+ was decided to be realized in steps, its commissioning being performed in parallel with scientific acquisition. Therefore, a shutdown is planned at the beginning of 2010 to install a new last stage of suspensions including monolithic arrangement of new mirrors.

The installation of monolithic suspensions will allow a relevant reduction of thermal noise level in the frequency region around 30-40 Hz. A new model [10] of the suspension noise has been developed to take into account the contribution to the noise due to the upper suspension stage, which becomes relevant since losses in fused silica wires are expected to be very low. Nevertheless, the gain in using the monolithic solution is evident (see figure 1). With the monolithic suspensions it is possible to extend the average sight distance for the BNS inspiral from $13 \mathrm{Mpc}$ up to $47 \mathrm{Mpc}$ and for the BBH inspiral from $44 \mathrm{Mpc}$ up to $394 \mathrm{Mpc}$, consequently increasing the observation rate up to $10^{-1} /$ year for BNS [9]. In order to have a fully monolithic stage, fused silica wires have to be properly attached to the mirror. This is possible by means of suitable "ears" silicate bonded to the mirror sides. Moreover, they have to be clamped in a proper way to the upper stage of suspension. These simple requests lead to some critical technical point:

- thin silica wires produced from pure material must have a diameter of the order of hundreds of micronsł. The working load for such wires is in the range of GPa, while

$\ddagger$ The suspension wires optimal diameter results as a compromise between the optimization of the dilution factor and the limit imposed by the breaking load. Moreover, requests on violin and bouncing modes frequencies are set by control issues, thus for Virgo + , the chosen wires diameter is $280 \mu \mathrm{m}$. 
for commercially available Suprasil ${ }^{\circledR}$ silica [11] the reported breaking stress is of the order of $50 \mathrm{MPa}$. Therefore, silica must be annealed to increase the maximum load to GPa. Also, after the production, one must avoid that anything touches the wires, since any scratch on them will abruptly decrease their breaking stress.

- suspension wires geometrical and dynamical parameters determine the statics of the suspended masses and the mirrors position control strategy§. Therefore, each of these parameters should be kept within a specified range.

- ears-to-mirrors and wires-to-ears connections must be not only easily operated and quality-controlled, but they must allow an efficient recovery action in case of failure. Further, they have not to spoil the silica wires performances in terms of low mechanical loss and high breaking stress. The same can be stated for the connections among the wires and the clamps on the payload.

- the payload recoil mass must be made of a dielectric material, in order to avoid eddy currents and magnetization effects due to the actuation coils.

\section{Realization of a monolithic suspension}

Silica parts composing the monolithic suspension can be joined using two methods, welding and chemical bonding. Welding is performed by locally heating the parts; bonding requires that a specific chemical technique, called silicate bonding $[3,12,13]$, be operated on very flat silica surfaces ( $\frac{\lambda}{10}$ to $\frac{\lambda}{4}$ depending on the process) to be glued together. Experience was collected that welding can be hardly performed in the neighborhood of a silicate bonded surface; otherwise, the bonded surface is deformed by the heat flux and the bonding strength is compromised. As a consequence, in some cases, direct welding of wires to ears, bonded to the masses, can be troublesome. Moreover, the latter technique makes difficult to recover the arrangement in case of wire failure.

The solution adopted for Virgo+ is a three steps operation, conceived for avoiding the use of welding tools in the vicinity of bonded ears. Referring to figure 2, the steps are:

- mirror is shaped by machining two flats on its curved sides. On the flats, two silica ears are silicate bonded, with grooves for the insertion of suspension elements (figure $2(\mathrm{a})$ ). The large bonding surface assures an adequate shear strength.

- a silica wire is produced with the lower tip welded to a silica piece named anchor, while the upper end is welded to a silica cone (figure 2 (b)). Each wire is tested to check for its strength and compliance to specifications.

- four wires are placed on the mirror by inserting the anchor under the bonded ear, within the groove, and silicate bonding the anchor to the ear (figure 2 (c)). Anchor top surface is optically flattened to allow silicate bonding. The upper cones of the wires are clamped to the payload making use of tight steel clamps (figure 2 (d)).

$\S$ The geometry of the wires should be optimized in order to minimize the angular-to-translation couplings, thus simplifying the control issues (see section 3.2.3). 


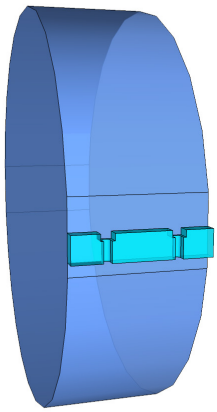

(a)

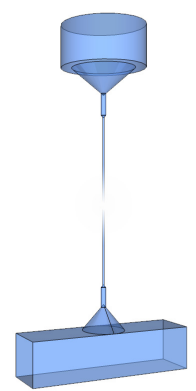

(b)

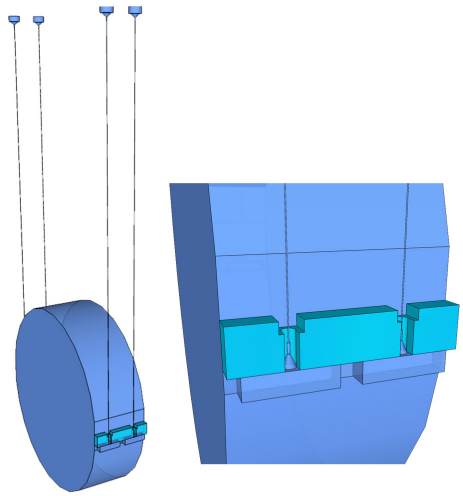

(c)

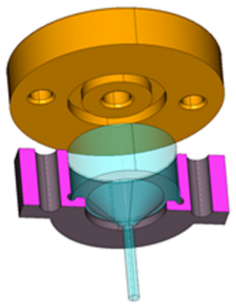

(d)

Figure 2. Sketch of the steps followed to realize a monolithic suspension, as detailed in the text.

The last two steps are performed in a clean chamber, at different levels of cleanliness up to 100 class around the mirror. Once the mirror is successfully suspended, it is safely blocked and the complete stage is assembled, including the reference mass.

\subsection{Mirrors preparation}

Virgo + mirrors are $100 \mathrm{~mm}$ thick with a diameter of $350 \mathrm{~mm}$, and a weight of 20.3 $\mathrm{kg}$. Mirrors are prepared in LMA Lyon clean rooms [14]. After the coating procedure the lateral flats are cleaned and the silica supports (ears) are glued with the silicate bonding technique ( $\mathrm{KOH}$ in $\mathrm{H}_{2} \mathrm{O}$ with a concentration of 1:250). Four magnets are glued on silica cylindrical supports through the cerama bond, then the supports are placed on the mirror surface with the anti-reflective coating through the silicate bonding. On Virgo + mirrors the markers are not used any more. After the glueing procedure the mirrors are stored in the LMA cleaning room for one month.

\subsection{Wires production and test}

Silica wires, welded to anchors and cones as previously described, are produced and validated in a three tasks pipeline.

3.2.1. Wire pulling with $\mathrm{CO}_{2}$ laser machine Silica wires are produced starting from high purity, $1.5 \mathrm{~mm}$ thick fused silica cylindrical bars commercially available (suitable material is Suprasil $\left.{ }^{\circledR}\right)$. Wires are obtained by melting the central region of a bar and subsequently pulling the two ends apart. In order to get reproducible results, the pulling has to be done in a controlled way. A machine for pulling fused silica wires using a 100 $\mathrm{W} \mathrm{CO}_{2}$ laser, developed at the University of Glasgow [15], has been assembled in a dedicated laser room at the site of the Virgo experiment. A schematic view of the functional parts of the machine is given in figure 3. Consider a silica rod clamped as in 


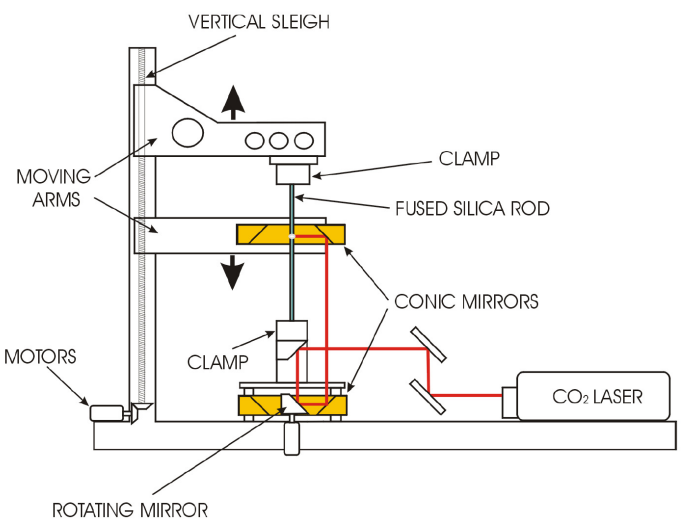

Figure 3. Schematic view of the $\mathrm{CO}_{2}$ laser machine employed for pulling the silica wires.

figure. The laser beam is delivered onto the fused silica rod by a series of gold coated mirrors. Thanks to the rotating and conic mirrors, the beam hit the rod continuously all around its axis, assuring an homogeneous local heating. The machine includes two arms connected to vertical sleighs which are moved by software controlled motors. Once the rod is melt by the laser, the upper arm pulls it upward following a given velocity profile and the wire is produced. The geometry of the pulled wire depends critically on the chosen velocity profile. Since the melting involves a small volume of the rod, from which the material is driven away, new material must be melt in order to feed the wire. Therefore, during the operation, the melting point must be shifted downward, and this is achieved by moving the conic mirror fixed to the lower arm. The machine can also be used for local welding, in which case it can be manually operated to get accurate results.

For the integration in the monolithic design, first of all a silica rod is welded at the ends to a silica anchor and to a silica cone. Then, the rod is annealed to melt structural imperfections and cracks. Finally, the wire is pulled from the rod as described. The profile of the obtained wire is very reproducible (within $10 \mu \mathrm{m}$ ), and its diameter $(280 \mu \mathrm{m})$ is remarkably constant along the wire, except at the ends where a conic neck is formed.

3.2.2. Strength test As soon as the wire is produced, it undergoes a safety load test. Being the working load about $5 \mathrm{~kg}$, all the wires are tested by holding $10 \mathrm{~kg}$ for $30 \mathrm{~min}$; if this test is successful, the wire is validated and passes to the following step.

The relevancy of this test is critical. Our experience proved that, if the pulling procedure is correctly followed, wire failure at the load test is very unlikely.

3.2.3. Geometrical and dynamical characterization Mirrors statics needs and control issues directly fix requests on the wire geometry and dynamics. At low frequencies, the 


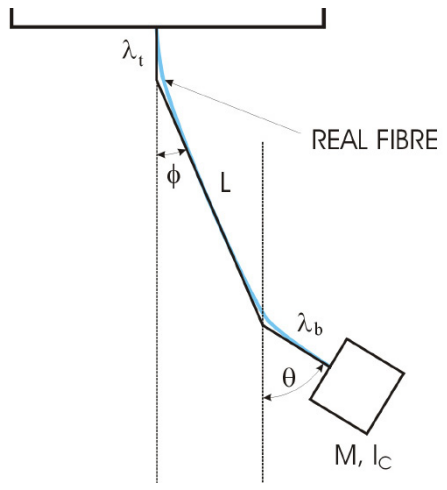

Figure 4. Scheme of the three-segment model of a silica wire. $\mathrm{M}$ and $\mathrm{I}_{C}$ are respectively the mass and the moment of inertia of the suspended mass. The model includes two angles $\theta$ and $\phi$, describing the two low frequency resonances of the wire pendulum motion.

dynamical behaviour of the suspending wire $\|$ is simply represented by a three-segment model [16] (see figure 4) whose parameters are the two lengths $\lambda_{t}$ and $\lambda_{b}$, called bending lengths, and the distance $L$ among these points, when the wire is loaded. An important request is that both the top and bottom bending points must lie on the horizontal plane of the center of mass, respectively of the payload marionette and of the suspended mass; in fact, the mirror longitudinal to pitch coupling is minimized in this condition. In addition, the first violin frequency $f_{V}$ and the bouncing mode frequency $f_{B}$ of the wire, both depending on the load, must match the Virgo+ specifications. Hence, after the strength test, the wire length is accurately measured. A specific tool, developed at INFN laboratory in Firenze $[16,17]$, allows the wire bending lengths to be determined within $0.1 \mathrm{~mm}$ accuracy. Also $f_{V}$ and $f_{B}$ are measured while the wire is suspended to this tool.

\subsection{The payload and assembling structure}

The monolithic suspension payload is composed by the new design marionette and recoil mass (RM), which inherit all the specifications given by the Virgo style payload [18], including the setup needed for the mirror local control purposes. Moreover, it must follow the requirements to ease the fused silica fibers installation and to avoid their breaking during the assembly and operation phases. For this reason the amagnetic stainless steel marionette has four cuts to allow the lateral insertion of the wires, and the holes, where the wires pass through, are widened to reduce the possibility of contact with the marionette body. The marionette arms are made of Tecapeek ${ }^{\circledR}$, a dielectric material, to remove the eddy current and magnetization effects due to the coils.

The new RM is designed to surround the mirror and protect it in case of suspension failure, and also to support the coils which act on the magnets glued on the mirror. A \| Remember that, due to the presence of the necks, the wire cannot be simply modeled as a cylinder. 

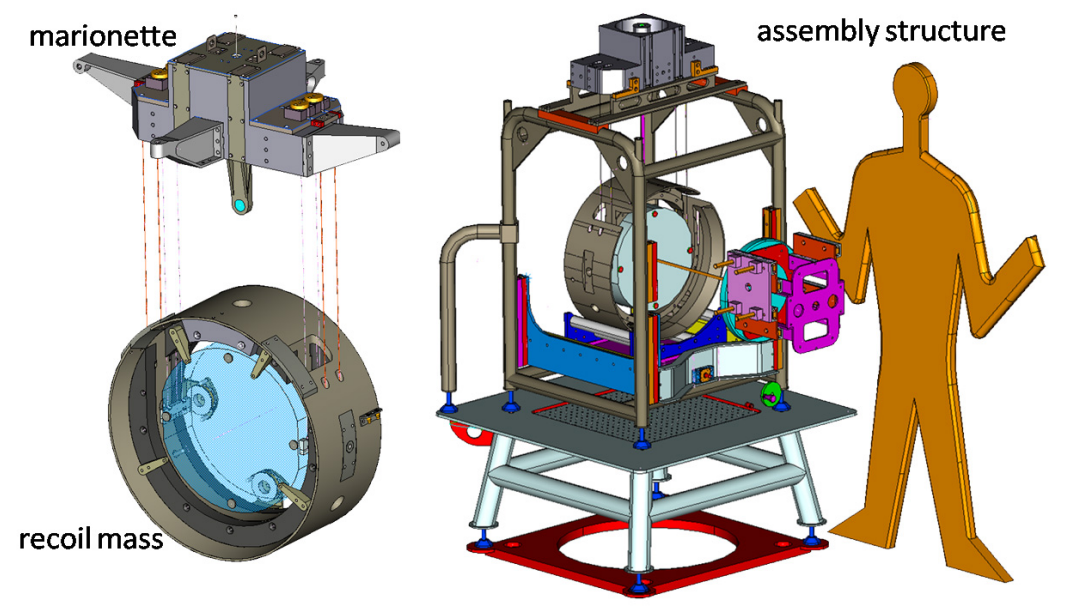

Figure 5. Sketch of the MS payload and of the assembly structure.

relevant change that has been implemented is to position the markers for the local control on the coil supports of the reaction mass. The new RM is composed by two main parts tightly fastened together to form a single stiff piece: an inner structure made of the dielectric Tecapeek ${ }^{\circledR}$ CF30 and an outer structure made of AISI316L steel. The aim is to remove the eddy current effects between the magnets, the coils and the RM body and to keep its stiffness and the weight similar to the present Virgo one (see figure 5). The RM design includes a small lateral mirror used for an optical lever which monitors the lateral position.

The assembly structure (see figure 5) is designed to ease the transportation and insertion in the tower of the whole monolithic suspension. The design follows all the requirements for positioning and handling all the payload pieces, starting from the clean room until when the payload is hung to the superattenuator. The most delicate phase of the payload assembly is when each silica fiber is transported and fixed to the marionette. Once all the payload is assembled, every component is blocked: in particular the mirror is blocked to its RM with the safety stops, and to the structure by a vacuum lid on its front side which protects it also from the pollution during the transportation.

\subsection{Dissipation measurements}

Table 1-left shows a summary of the expected and measured quality factors on a 1:1 scale dummy monolithic suspension realized in a vacuum chamber. The quality factor of the pendulum mode $(0.58 \mathrm{~Hz})$ appears to be lower than the expected one. For this reason a series of tests has been made to understand the dominant losses in the measurement. Possible loss sources can be introduced by the upper cone clamping system, the anchor to ear bonding, the recoil of the suspension structure or other frictional processes affecting the system. 
The monolithic suspensions for the interferometer Virgo

Table 1. (Left) Measured and theoretical quality factors for the monolithic dummy suspension $(22 \mathrm{Kg})$. (Right) Measured and theoretical quality factors for one silica wire loaded with $1 \mathrm{~kg}$.

\begin{tabular}{ll}
\hline$Q_{\text {PendulumMeas }}$ & $2.5 \cdot 10^{6}$ \\
\hline$Q_{\text {PendulumTheor }}$ & $2 \cdot 10^{9}$ \\
$Q_{\text {RecoilTheor }}$ & $4 \cdot 10^{7}$ \\
$Q_{\text {Gas }}$ & $9 \cdot 10^{7}$ \\
$Q_{\text {SilicateBonding }}$ & $3 \cdot 10^{8}$ \\
\hline
\end{tabular}

\begin{tabular}{ll}
\hline$Q_{\text {PendulumMeas }}$ & $6 \cdot 10^{7}$ \\
\hline$Q_{\text {PendulumTheor }}$ & $9 \cdot 10^{8}$ \\
$Q_{\text {RecoilTheor }}$ & $8 \cdot 10^{8}$ \\
$Q_{\text {Gas }}$ & $8 \cdot 10^{8}$ \\
\hline
\end{tabular}

3.4.1. Anchors bonding A FEM simulation was performed to evaluate the losses due to the bonded region of the ears. In the simulation the fraction of elastic energy lost in the bonded zone during the mirror oscillation was calculated. The result is a value of $Q_{\text {ears }}^{\text {pendulum }}=2.2 \times 10^{12}$ for the pendulum mode and $\phi_{\text {ears }}^{\text {drum }}=2.9 \times 10^{8}$ for the mirror drum mode [19]. As a consequence the losses due to the bonded region are negligible for both the suspension and mirror thermal noise.

3.4.2. Upper clamp To evaluate if the losses due to the upper clamp system were predominant, two tests were performed:

- a silica rod of $1.5 \mathrm{~mm}$ was welded to the upper clamp system and then put in a vacuum chamber. The $\mathrm{Q}$ measurements give a mean value of $Q=1 \cdot 10^{7}$. This value is the one expected for the silica rod.

- an upper clamp with a Virgo+ standard silica wire was used to suspend different weights to verify the behaviour of the pendulum quality factor. Table 1-right shows the results for $1 \mathrm{~kg}$. The measured values are dominated by recoil losses, as detailed in the following.

For the explored Q values no evidence of extra losses induced by the clamp were found.

3.4.3. Recoil losses Comparing the measurements in tables 1-left and 1-right, it is interesting to see that the quality factor of the pendulum mode decreases with the inverse ratio of the suspended masses. It seems to fit the recoil losses model.

The recoil losses are given by the following equation:

$$
Q_{\text {Rec }}=\frac{k l}{M g \theta}
$$

where $k$ is the spring constant of the structure (at the pendulum frequency), $\theta$ is the phase angle between the force applied to the structure and its displacement, $l$ is the length of the fiber loaded with the mass $M$. All these parameters are listed in [20]. The ratio between the two quality factor measurements is consistent with the inverse ratio of the masses of the two suspensions: $22 \mathrm{~kg}$ for the first suspension and $1 \mathrm{~kg}$ for the second. Other measurements with 3 and $5 \mathrm{~kg}$ are in course to verify if the $\mathrm{Q}$ scales linearly with the mass. 


\section{Conclusions}

Monolithic suspensions will reduce the suspension thermal noise at low frequency in Virgo+. The complete suspension stages are going to be installed in the interferometer at the beginning of 2010. The suspension design and the realization steps have been successfully validated; the developed technique allows the suspension to be easily realized and rapidly recovered in case of wire failure. In the new local control design, the markers have been moved from the mirrors to the recoil mass. An estimation of the suspension pendulum quality factor is currently ongoing in order to understand if losses introduced by the wires clamping system can be neglected; at present, the measurements are probably limited by recoil losses in the suspension support. The latter point will be carefully investigated by exploiting the dependence of the pendulum quality factor on the suspended mass.

\section{References}

[1] http://www.virgo.infn.it

[2] Cagnoli G et al 1999 Phys. Lett. A 255230

[3] Amico P et al 2002 Rev. Sci. Instrum. 73 3318-23

[4] Amico P et al 2001 Nucl. Instrum. Meth. A 461 297-99

[5] Lück H et al 2006 Class. Quantum Grav. 23 S71S78

[6] Cagnoli G et al 2002 Class. Quantum Grav. 19 4043-58

[7] The Virgo Collaboration 2009 Advanced Virgo Baseline Design availabe at http://wwwcascina.virgo.infn.it/advirgo/docs.html

[8] Tournefier E on behalf of the Virgo collaboration 2009 News from Virgo: present status and future upgrades Proceedings of the 21st Rencontres de Blois

[9] Puppo P 2009 Virgo note "Virgo+MS sensitivity curve" available at https : //tds.ego-gw.it/ql/?c=6957

[10] Piergiovanni F, Punturo M and Puppo P 2009 Virgo Document available at https : //tds. ego-gw. it/ql/?c=2199

[11] Trade mark from Heraeus Quarzglas http://www.heraeus-quarzglas.com

[12] Gwo D H 2001 Ultra precision and reliable bonding method United States Patent $\mathrm{n}^{\circ}$ US 6284085 B1

[13] Sneddon P H, Bull S, Cagnoli G, Crooks D R M, Elliffe E J, Faller J E, Fejer M M, Hough J and Rowan S 2003 Class. Quantum Grav. 20 5025-37

[14] http: //lma.in2p3.fr

[15] Heptonstall A, Martin I, Cumming A, Cantley C A, Cagnoli G, Jones R and Crooks D R M 2005 LIGO technical note available at http://www. ligo.caltech.edu/docs/T/T050206-00.pdf

[16] Piergiovanni F, Cagnoli G, Campagna E, Cesarini E, Lorenzini M, Losurdo G, Martelli M, Viceré A and Vetrano F 2009 Amaldi 8 poster available at http://sites.google.com/site/amaldi8posters1/posters/Poster_137_Piergiovanni.pdf

[17] Cagnoli G et al. (in preparation)

[18] Bernardini A, Majorana E, Puppo P, Rapagnani P, Ricci F and Testi G. 1999 Rev. Sci. Instrum. 703463

[19] Puppo P 2009 Virgo note "FEM of the strain energy stored in the Hydroxide-Catalysis Bonded ears of the mirrors for Virgo+" VIR-XXX-09 (in preparation)

[20] Cagnoli G, Gammaitoni L, Hough J, Kovalik J, McIntosh S, Punturo M and Rowan S 2000 Phys. Rev. Lett. 85 2442-45 